\title{
Dissipativity Theory for Switched Systems
}

\author{
Jun Zhao and David J. Hill, Fellow, IEEE
}

\begin{abstract}
A framework of dissipativity theory for switched systems using multiple storage functions and multiple supply rates is set up. Each subsystem of a switched system is associated with a storage function to describe the "energy" stored in the subsystem, and is associated with a supply rate that represents energy coming from outside the subsystem when the subsystem is active. The exchange of "energy" between the active subsystem and an inactive subsystem is characterized by cross-supply rates. Stability is reached when all supply rates can be made negative, as long as the total exchanged energy between the active subsystem and any inactive subsystems is finite in some sense. Two special forms of dissipativity, passivity and $L_{2}$-gain, are addressed. For both cases, asymptotic stability is guaranteed under certain "negative" output feedback plus asymptotic zero state detectability. Switched passivity conditions and switched $L_{2}$-gain inequalities are, respectively, derived, which are generalizations of classical ones. Feedback invariance of passivity and a small-gain theorem are also given.
\end{abstract}

Index Terms-Dissipativity, $L_{2}$-gain, passivity, stability, switched systems.

\section{INTRODUCTION}

D ISSIPATIVITY theory of nonlinear systems, which was developed by Willems [40] and further extended by Hill and Moylan [17]-[19], has become one of the major approaches to the study of complex systems, especially for the stabilization issue. Based on the consideration of abstract energy that may have certain physical meaning for a particular system, roughly speaking, dissipativity usually means that the increase in energy storage in the system is no more than the supplied energy from outside the system. Dissipativity is characterized by storage functions and supply rates, which represent the energy stored inside the system and energy supplied from outside the system, respectively.

Dissipativity is a desirable system property for two reasons. First, the storage functions induced by dissipativity usually provide natural candidates for Lyapunov functions. Therefore, in many cases, stability and stabilization problems can be solved once the dissipativity property is assured [3], [34], [41]. Such storage functions often come from simple observation of physi-

Manuscript received January 4, 2007; revised June 20, 2007 and August 7, 2007. Recommended by Associate Editor I. Kolmanovsky. This work was supported in part by the Australian Research Council Federation Fellowship and Centres of Excellence Schemes, in part by the National Natural Science Foundation (NNSF) of China under Grant 60574013, and in part by the Research Grants Council of Hong Kong.

J. Zhao is with the Key Laboratory of Integrated Automation of Process Industry, Ministry of Education of China, Northeastern University, Shenyang 110004, China. He is also with the Department of Information Engineering, Research School of Information Sciences and Engineering, The Australian National University, Canberra, A.C.T. 0200, Australia (e-mail: jun.zhao@anu.edu.au).

D. J. Hill is with the Department of Information Engineering, Research School of Information Sciences and Engineering, The Australian National University, Canberra, A.C.T. 0200, Australia (e-mail: david.hill@anu.edu.au).

Digital Object Identifier 10.1109/TAC.2008.920237 cal variables, or can be constructed intuitively. The other reason for dissipativity to be useful is that it can give rise to stabilization results of interconnected systems, and thus, qualifies as an efficient tool for the analysis and design of composite (largescale) systems. In this respect, the well-known invariance of passivity under feedback interconnection and small-gain theorems plays a central role. There are many papers concerning dissipativity and passivity-based control (see, for example, the results summarized in recent books [13], [26], [39]).

On the other hand, switched systems as an important class of hybrid systems have drawn considerable attention in recent years. This is mainly due to the switched and hybrid nature of many physical processes and the growing use of computers in the control of physical plants [5], [23], [24], [38]. The extensive engineering applications of switched systems are also motivated by the better performance that can be achieved using a controller switching strategy [20], [29], [36].

Stability issues are currently a major focus in studying switched systems. Though effective theory and approaches for the analysis and control of continuous-time or discrete-time systems have been available, and to a large extent, are welldeveloped, similar techniques for switched systems may not be valid due to the complicated behavior caused by interaction between continuous dynamics and discrete dynamics. Consequently, the study of stability for switched systems is more difficult than the study for continuous systems or discrete systems, and so becomes a challenging issue [5], [23], [28], [44]. In this respect, the Lyapunov stability theory and its variations or generalizations still play a dominating role [22], [24], [25], [38]. A common Lyapunov function for all subsystems guarantees stability under an arbitrary switching law [23]. Many methods have been proposed to construct such a common Lyapunov function (see, for instance, a hierarchical switching strategy [21] and a common quadratic Lyapunov function method [30]). Since most switched systems in practice do not possess a common Lyapunov function, more attention has been paid to stabilization under some properly chosen switching law. The multiple Lyapunov function technique, proposed by Peleties and DeCarlo [31], and further extended by Branicky [2] is a powerful tool for finding such a switching law or identifying a class of useful switching laws. Many results in this direction have been available (for example, see [12], [23], and [24] and recent work on output feedback control [7], predictive control [27], and $H_{\infty}$ control [43], [45]).

Given the importance of dissipativity concepts for smooth systems, it is expected that they are also useful for hybrid and switched systems. This has not received much attention until now with few results appearing on the topic. Dissipativity and stability analysis for general impulsive and hybrid systems were addressed in [8] and [9], where necessary and sufficient conditions for dissipativity using a common storage function 
and a common supply rate were established. Since switched systems are a special class of impulsive systems, these conditions also provide systematic and effective tests for dissipativity and stability of switched systems. Recently, these results were generalized as a vector version of dissipativity theory for large-scale impulsive dynamical systems [10]. A novel class of energy-based hybrid controllers was proposed to achieve enhanced energy dissipation [11]. Passivity of nonlinear control systems based on completeness and using controller switching was discussed in [33]. A passivity-based design method for switched control systems was proposed in [4]. Passivity analysis of discrete-time hybrid systems was carried out in [1]. $L_{2}$-induced gains and root-mean-square gains were introduced in [15] and [16], respectively, and conditions for all subsystems to share the same gain were derived. An average dwell time method was proposed for stability and $L_{2}$-gain analysis of delay switched systems [37]. Besides, there are applications of passivity-based control to electrical systems with a hybrid nature. For example, the hybrid passivity idea was applied to a three-phase voltage-sourced reversible-boost-type rectifier [6], and a passivity-based control strategy was proposed for switched reluctance motors with nonlinear magnetic circuits [32]. An energy-based switching control strategy was presented to globally stabilize the cart-pendulum system [47]. However, all of the results mentioned earlier adopt a unified "storage function" to characterize dissipativity or passivity. Actually, dissipativity associates a particular mathematical description with a set of closely related storage functions corresponding to possible physical realizations [17].

The adoption of multiple supply rates and multiple storage functions for switched systems is natural. This is not only for the purpose of relaxing the restriction of single storage functions, but also based on practical considerations. Indeed, each subsystem usually has its own storage function according to certain physical meaning, and in fact, different subsystems may have completely different physical meanings. Similarly, supply rates may sensibly vary across the subsystems. From this point of view, the dissipativity description for a switched system using the same storage function and the same supply rate for all subsystems is often unreasonable and impractical. Individual features of storage functions and supply rates should be preserved in a proper description of dissipativity.

Theoretically, since storage functions often qualify as Lyapunov function candidates, the demand for multiple Lyapunov functions in the study of switched systems naturally leads to the adoption of multiple storage functions in the dissipativity description, which, in turn, needs multiple supply rates. Thus, from physical and theoretical viewpoints, it is clear that the classical notion of dissipativity needs to be cleared to cover hybrid and switching settings. Zefran et al. [42] proposed a notion of passivity by using multiple storage functions. Stability and feedback invariance were proven. However, this passivity concept requires each storage function to be nonincreasing on the switching sequence of consecutive "switched on" times as a prerequisite to meet Branicky's nonincreasing condition of multiple Lyapunov functions, which, in turn, guarantees stability. Besides, no asymptotic stability is induced by strict pas- sivity or feedback passivity. Multiple storage functions were also exploited to study a passivity property of switched systems with state-dependent switching in [46]. However, this passivity property is based on certain constraints directly put on storage functions of inactive subsystems. Some additional conditions are needed to deduce stability.

This paper presents a framework of dissipativity for switched systems using multiple storage functions and multiple supply rates. Unlike continuous systems, a switched system has an unusual phenomenon that must be taken into consideration when dealing with change of energy. A storage function of a subsystem is still "changing" on the time intervals when the subsystem is inactive. For example, this can simply be because all subsystems share the same state variables. The active subsystem drives the state which, in turn, causes the change of storage functions of inactive subsystems. This "changing" energy of any inactive subsystem, though not necessarily real energy, is viewed as "exported energy" from the active subsystem, and is characterized by cross-supply rates.

The organization of the paper is as follows. Section II gives the preliminaries. In Section III, we present a dissipativity notion for switched systems using multiple storage functions and multiple supply rates. Associated properties are also discussed, focusing on stability. Sections IV and V briefly address two special forms of dissipativity, namely, passivity and $L_{2}$-gain, respectively, proving a passivity theorem and small-gain theorem. Finally, Section VI contains the concluding remarks.

\section{PRELIMINARIES}

In this paper, we consider a switched system of the form

$$
\begin{aligned}
& \dot{x}=f_{\sigma}\left(x, u_{\sigma}\right) \\
& y=h_{\sigma}(x)
\end{aligned}
$$

where $\sigma$ is the switching signal taking values in

$$
M=\{1,2, \ldots, m\}
$$

which may depend on time or state, or both, or even be generated by higher level hybrid feedback in the loop; $x \in R^{n}$ is the state, $u_{i}$ and $h_{i}(x)$ are the input and output vectors of the $i$ th subsystem, respectively. Further, $f_{i}(x, u)$ and $h_{i}(x)$ are continuous and satisfy $f_{i}(0,0)=0$ and $h_{i}(0)=0, i=1,2, \ldots, m$. Here, we apply the standard notations adopted from [2] and [24]. The switching signal $\sigma$ can be characterized by the switching sequence

$$
\Sigma=\left\{x_{0} ;\left(i_{0}, t_{0}\right),\left(i_{1}, t_{1}\right), \ldots,\left(i_{n}, t_{n}\right), \ldots \mid i_{n} \in M, n \in N\right\}
$$

in which $t_{0}$ is the initial time, $x_{0}$ is the initial state, and $N$ is the set of nonnegative integers. When $t \in\left[t_{k}, t_{k+1}\right), \sigma(t)=i_{k}$, that is, the $i_{k}$ th subsystem is active. Therefore, the trajectory $x(t)$ of the switched system (1) is defined as the trajectory $x_{i_{k}}(t)$ of the $i_{k}$ th subsystem when $t \in\left[t_{k}, t_{k+1}\right)$. We assume that the state of the switched system (1) does not jump at the switching instants, i.e., the trajectory $x(t)$ is everywhere continuous. The switching sequence $\Sigma$ may or may not be infinite. In the finite case, we may take $t_{n+1}=\infty$, with all further definitions and results still valid. 


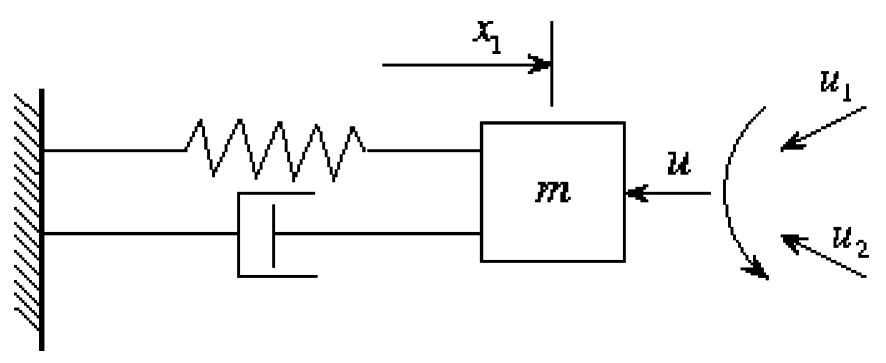

Fig. 1. Mass-spring-damper system with controller switching.

For any $j \in M$, let

$$
\Sigma \mid j=\left\{t_{j_{1}}, t_{j_{2}}, \ldots, t_{j_{n}}, \ldots, i_{j_{q}}=j, q \in N\right\}
$$

be the sequence of switching times when the $j$ th subsystem is switched ON, and thus

$$
\left\{t_{j_{1}+1}, t_{j_{2}+1}, \ldots, t_{j_{n}+1}, \ldots, i_{j_{q}}=j, q \in N\right\}
$$

is the sequence of switching times when the $j$ th subsystem is switched OFF.

Assumption 2.1: For any finite $T>t_{0}$, there exists a positive integer $K_{T}$, which may depend on $T$, such that during the time interval $\left[t_{0}, T\right]$ the system (1) switches no more than $K_{T}$ times.

As usually assumed in the literature, this assumption simply means that only finite many switchings occur during any finite time and the number of switchings is bounded from above by an integer that depends on the finite time. This assumption is very general because otherwise the switching can be arbitrarily fast in some finite time interval, which is obviously unacceptable in practice.

By $L_{1}[0, \infty)$ we denote the usual $L_{1}$ function space over $[0, \infty)$, that is, $\mu=\mu(t) \in L_{1}[0, \infty)$ if

$$
\int_{0}^{\infty}|\mu(t)| d t<\infty .
$$

Let $L_{1}^{+}[0, \infty)$ denote the subset of $L_{1}[0, \infty)$ consisting of all nonnegative functions.

\section{DISSIPATIVITY}

This section gives the description of dissipativity for switched systems by using multiple storage functions and multiple supply rates, and studies related properties focusing on stability. The key point here is to handle the difficulty caused by the change of storage functions when corresponding subsystems are inactive.

\section{A. Motivating Examples}

We examine two examples.

Example 3.1: [35]. Consider a mechanical system with controller switching depicted in Fig. 1.

The mass-spring-damper system with nonlinear stiffness and damping is described by the following nonlinear system

$$
\begin{aligned}
\dot{x}_{1} & =x_{2} \\
\dot{x}_{2} & =-\frac{1}{m} f\left(x_{1}\right)-\frac{1}{m} g\left(x_{2}\right)+\frac{1}{m} u \\
y & =x_{2}
\end{aligned}
$$

where $f(0)=0, g(0)=0$.

Suppose that we are only allowed to apply two prespecified candidate controllers

$$
u_{i}=-\Delta f_{i}\left(x_{1}\right)-\Delta g_{i}\left(x_{2}\right)+v_{i}, i=1,2
$$

with

$$
\left(g\left(x_{2}\right)+\Delta g_{i}\left(x_{2}\right)\right) x_{2} \geq 0
$$

$\Delta f_{i}(0)=0$, and $\Delta g_{i}(0)=0$ to the system and switch between them. This results in the switched system

$$
\begin{aligned}
\dot{x}_{1}= & x_{2} \\
\dot{x}_{2}= & -\frac{1}{m}\left(f\left(x_{1}\right)+\Delta f_{i}\left(x_{1}\right)\right) \\
& -\frac{1}{m}\left(g\left(x_{2}\right)+\Delta g_{i}\left(x_{2}\right)\right)+\frac{1}{m} v_{i} \\
y= & x_{2}
\end{aligned}
$$

which may be considered as a mechanical system with changing stiffness and damper, or changing environment [42]. It is well known that, for each $i$ and fixed $\Delta f_{i}, \Delta g_{i}$, the $i$ th subsystem is passive from the input $v_{i}$ to the output $x_{2}$.

The energy functions of the two subsystems are

$$
S_{i}(x)=\int_{0}^{x_{1}}\left(f(s)+\Delta f_{i}(s)\right) d s+\frac{1}{2} m x_{2}^{2}, i=1,2 .
$$

When the first subsystem is active on $\left[T_{1}, T_{2}\right)$ we have

$$
S_{1}\left(x\left(T_{2}\right)\right)-S_{1}\left(x\left(T_{1}\right)\right) \leq \int_{T_{1}}^{T_{2}} \omega_{1}^{1}\left(v_{1}(\tau), y(\tau)\right) d \tau
$$

with

$$
\omega_{1}^{1}\left(v_{1}, y\right)=v_{1} y
$$

and the change of the energy function for the second subsystem is given by

$$
S_{2}\left(x\left(T_{2}\right)\right)-S_{2}\left(x\left(T_{1}\right)\right) \leq \int_{T_{1}}^{T_{2}} \omega_{2}^{1}\left(x(\tau), v_{1}(\tau), y(\tau), \tau\right) d \tau
$$

with

$$
\omega_{2}^{1}\left(x, v_{1}, y, t\right)=v_{1} y+\left(\Delta f_{2}\left(x_{1}\right)-\Delta f_{1}\left(x_{1}\right)\right) x_{2} .
$$

Similarly, when the second subsystem is active on $\left[T_{1}, T_{2}\right)$, we have

$$
S_{2}\left(x\left(T_{2}\right)\right)-S_{2}\left(x\left(T_{1}\right)\right) \leq \int_{T_{1}}^{T_{2}} \omega_{2}^{2}\left(v_{2}(\tau), y(\tau)\right) d \tau
$$

with

$$
\omega_{2}^{2}\left(v_{2}, y\right)=v_{2} y
$$

and the change of the energy function for the first subsystem is given by

$$
\begin{aligned}
& S_{1}\left(x\left(T_{2}\right)\right)-S_{1}\left(x\left(T_{1}\right)\right) \\
& \quad \leq \int_{T_{1}}^{T_{2}} \omega_{1}^{2}\left(x(\tau), v_{2}(\tau), y(\tau), \tau\right) d \tau
\end{aligned}
$$


with

$$
\omega_{1}^{2}\left(x, v_{2}, y, t\right)=v_{2} y+\left(\Delta f_{1}\left(x_{1}\right)-\Delta f_{2}\left(x_{1}\right)\right) x_{2} .
$$

Example 3.2: Consider the switched system

$$
\begin{gathered}
\left(\begin{array}{c}
\dot{x}_{1} \\
\dot{x}_{2}
\end{array}\right)=f_{\sigma}\left(x_{1}, x_{2}, u_{\sigma}\right) \\
y=h_{\sigma}\left(x_{1}, x_{2}\right)
\end{gathered}
$$

where

$$
\begin{gathered}
f_{1}\left(x_{1}, x_{2}, u_{1}\right)=\left(\begin{array}{c}
x_{2} \\
-x_{1}^{3}-x_{2}+u_{1}
\end{array}\right) \\
f_{2}\left(x_{1}, x_{2}, u_{2}\right)=\left(\begin{array}{c}
x_{2} \\
\left(1+x_{2}^{2}\right)\left(-x_{1}-2 x_{2}+u_{2}\right)
\end{array}\right)
\end{gathered}
$$

and

$$
h_{1}\left(x_{1}, x_{2}\right)=h_{2}\left(x_{1}, x_{2}\right)=x_{2} \text {. }
$$

Choose

$$
S_{1}(x)=\frac{1}{4} x_{1}^{4}+\frac{1}{2} x_{2}^{2}
$$

and

$$
S_{2}(x)=\frac{1}{2} x_{1}^{2}+\frac{1}{2} \ln \left(1+x_{2}^{2}\right) .
$$

When the first subsystem is active on $\left[T_{1}, T_{2}\right)$, we have

$$
S_{1}\left(x\left(T_{2}\right)\right)-S_{1}\left(x\left(T_{1}\right)\right) \leq \int_{T_{1}}^{T_{2}} \omega_{1}^{1}\left(u_{1}(\tau), y(\tau)\right) d \tau
$$

with

$$
\omega_{1}^{1}\left(u_{1}, y\right)=u_{1} y
$$

and

$$
\begin{aligned}
& S_{2}\left(x\left(T_{2}\right)\right)-S_{2}\left(x\left(T_{1}\right)\right) \\
& \quad \leq \int_{T_{1}}^{T_{2}} \omega_{2}^{1}\left(x(\tau), u_{1}(\tau), h_{1}(x(\tau)), \tau\right) d \tau
\end{aligned}
$$

with

$$
\omega_{2}^{1}\left(x, u_{1}, h_{1}, t\right)=x_{2}\left(x_{1}-\frac{x_{1}^{3}}{1+x_{2}^{2}}\right)+\frac{x_{2}}{1+x_{2}^{2}} u_{1} .
$$

Similarly, when the second subsystem is active on $\left[T_{1}, T_{2}\right)$, we have

$$
S_{2}\left(x\left(T_{2}\right)\right)-S_{2}\left(x\left(T_{1}\right)\right) \leq \int_{T_{1}}^{T_{2}} \omega_{2}^{2}\left(u_{2}(\tau), y(\tau)\right) d \tau
$$

with

$$
\omega_{2}^{2}\left(u_{2}, y\right)=u_{2} y
$$

and

$$
\begin{aligned}
& S_{1}(\left.x\left(T_{2}\right)\right)-S_{1}\left(x\left(T_{1}\right)\right) \\
& \quad \leq \int_{T_{1}}^{T_{2}} \omega_{1}^{2}\left(x(\tau), u_{2}(\tau), h_{2}(x(\tau)), \tau\right) d \tau
\end{aligned}
$$

with

$$
\begin{aligned}
& \omega_{1}^{2}\left(x, u_{2}, h_{2}, t\right) \\
& \quad=\left(1+x_{2}^{2}\right) x_{2}\left(\frac{x_{1}^{3}}{1+x_{2}^{2}}-x_{1}\right)+x_{2}\left(1+x_{2}^{2}\right) u_{2} .
\end{aligned}
$$

In these examples, the change of energy can be categorized into two types: first, corresponding to an active subsystem, the change of energy is characterized by $\omega_{i}^{i}$, which is exactly the same as the usual supply rates of passivity, and the other is for inactive systems, determined by $\omega_{i}^{j}, i \neq j$.

\section{B. Definition of Dissipativity}

Recall that the dissipativity property in state space form for a continuous control system

$$
\begin{aligned}
& \dot{x}=f(x, u) \\
& y=h(x)
\end{aligned}
$$

is characterized by a supply rate function $\omega(\cdot, \cdot)$ and a storage function $S(x)$ satisfying

$$
S(x(t))-S\left(x\left(t_{0}\right)\right) \leq \int_{t_{0}}^{t} \omega(u(\tau), y(\tau)) d \tau
$$

for $\forall t \geq t_{0}$ (see [40]).

This classical form of dissipativity is obviously applicable to the switched system (1) as

$$
S(x(t))-S\left(x\left(t_{0}\right)\right) \leq \int_{t_{0}}^{t} \omega\left(u_{\sigma(\tau)}(\tau), h_{\sigma(\tau)}(x(\tau))\right) d \tau .
$$

However, this property is restrictive for switched systems because, as seen in the earlier examples, each subsystem usually has its individual supply rate $\omega_{i}$, and thus, a storage function $S_{i}(x)$ when this subsystem is active. A common supply rate $\omega$, and thus, a common storage function $S(x)$ for all subsystems may be difficult to find or may not exist at all. Therefore, it is reasonable and necessary to adopt multiple supply rates and multiple storage functions to characterize the dissipativity property for switched systems. However, a simple adoption of multiple storage functions and supply rates of each subsystem may result in the loss of a desirable property that is expected to be induced by dissipativity, namely, stability. This may happen mainly because of the negative impact of inactive subsystems on the behavior of the whole switched system. The major difficulty here is that unlike multiple Lyapunov functions, where a nonincreasing condition on a "switched ON" time sequence is a basic assumption though the Lyapunov function is allowed to increase when the corresponding subsystem is inactive, storage functions are allowed to increase not only on time intervals when the corresponding subsystems are inactive but also on the "switched ON" time sequence. Therefore, the change of storage functions must be carefully taken into account when the corresponding subsystems are inactive.

Definition 3.3: System (1) is said to be dissipative under the switching law $\Sigma$ if there exist positive-definite continuous functions $S_{1}(x), S_{2}(x), \ldots, S_{m}(x)$, called storage functions, locally integrable functions $\omega_{i}^{i}\left(u_{i}, h_{i}\right), \quad 1 \leq i \leq m$, called supply 
rates, and locally integrable functions $\omega_{j}^{i}\left(x, u_{i}, h_{i}, t\right), \quad 1 \leq$ $i, j \leq m, i \neq j$, called cross-supply rates, such that

$$
\begin{aligned}
& S_{i_{k}}(x(t))-S_{i_{k}}(x(s)) \\
& \quad \leq \int_{s}^{t} \omega_{i_{k}}^{i_{k}}\left(u_{i_{k}}(\tau), h_{i_{k}}(x(\tau))\right) d \tau \\
& \quad k=0,1,2, \ldots, t_{k} \leq s \leq t<t_{k+1} \\
& S_{j}(x(t))-S_{j}(x(s)) \\
& \quad \leq \int_{s}^{t} \omega_{j}^{i_{k}}\left(x(\tau), u_{i_{k}}(\tau), h_{i_{k}}(x(\tau)), \tau\right) d \tau \\
& \quad j \neq i_{k}, k=0,1,2, \ldots, t_{k} \leq s \leq t<t_{k+1} .
\end{aligned}
$$$$
\text { i) }
$$$$
\text { ii) }
$$

iii) For any $i, j$, there exist $u_{i}(t)=\alpha_{i}(x(t), t)$ and $\phi_{j}^{i}(t) \in$ $L_{1}^{+}[0, \infty)$, which may depend on $u_{i}$ and the switching sequence $\Sigma$, such that

$$
\begin{aligned}
& f_{i}\left(0, \alpha_{i}(0, t)\right) \equiv 0, \quad \forall t \geq t_{0} \\
& \omega_{i}^{i}\left(u_{i}(t), h_{i}(x(t))\right) \leq 0, \quad \forall t \geq t_{0}
\end{aligned}
$$

and

$$
\omega_{j}^{i}\left(x(t), u_{i}(t), h_{i}(x(t)), t\right)-\phi_{j}^{i}(t) \leq 0, \quad \forall j \neq i, \forall t \geq t_{0} .
$$

Remark 3.4: In Definition 3.3, $S_{j}$ and $\omega_{j}^{j}$ are the usual storage function and supply rate, respectively, for the $j$ th subsystem when active. Unlike in the classical definition of dissipativity where storage functions are semipositive definite, we need positive definite storage functions here for switched systems to induce stability and output stabilizability. This situation is the same as that of multiple Lyapunov functions [2], where all Lyapunov functions for subsystems need to be positive definite. It is worthwhile noticing that the $j$ th subsystem is inactive on the time interval $\left[t_{k}, t_{k+1}\right)$, and thus, the "energy" $S_{j}(x)$ may apparently remain unchanged. However, because all subsystems share the same state variable, $S_{j}(x)$ indeed changes from $S_{j}\left(x\left(t_{k}\right)\right)$ to $S_{j}\left(x\left(t_{k+1}\right)\right)$. This can be viewed as the result of "imported energy" from the active $i_{k}$ th subsystem into the inactive $j$ th subsystem. This "energy" is characterized by the "cross-supply rate" $\omega_{j}^{i}$ from the $i$ th subsystem to the $j$ th subsystem and satisfies the dissipation inequalities (18).

In Condition iii), (19) is needed to guarantee that the origin is the equilibrium of the system (1) with the control $u_{i}(t)$. Equation (19) is obviously satisfied for a broad class of controllers. For example, any state feedback controller $u_{i}=\alpha_{i}(x)$ with $\alpha_{i}(0)=$ 0 satisfies (19). Equations (20) and (21) simply mean that, for at least one $u_{i}(t)$, if no external energy is supplied to the $i$ th subsystem when active, then the total "energy" coming from the active $i$ th subsystem to the inactive $j$ th subsystem is finite. This condition is natural and reasonable. Otherwise, infinitely large energy would be produced by the $i$ th subsystem without external energy. When the cross-supply rates are of the form

$$
\omega_{j}^{i}\left(x, u_{i}, h_{i}, t\right)=\vartheta_{j}^{i}(x) \omega_{i}^{i}\left(u_{i}, h_{i}\right)+\phi_{j}^{i}(t)
$$

with positive functions $\vartheta_{j}^{i}(x)$ and $\phi_{j}^{i}(t) \in L_{1}^{+}[0, \infty)$, Condition iii) is automatically satisfied. In the sequel, for simplicity, we assume that any $u_{i}(t)$ satisfying (19) and (20) also satisfies (21).

Though all subsystems are assumed to be time invariant, the overall switched system will have time-variant features because of switching. This is even more so for the case of time-dependent switching laws. Considering this, the cross-supply rates are defined to be time dependent to cover more general situations. Such time-variant cross-supply rates also indicate that on different active time intervals of the $i$ th subsystem, the supply rate of the energy from $i$ th subsystem to $j$ th subsystem may be different.

Remark 3.5: When i) holds for a common storage function $S_{i}(x)=S(x)$ and a common supply rate

$$
\omega_{i}^{i}\left(u_{i}, h_{i}\right)=\omega\left(u_{i}, h_{i}\right)
$$

with $\omega\left(0, h_{i}\right) \leq 0$, ii) is satisfied with

$$
\omega_{j}^{i}\left(x, u_{i}, h_{i}, t\right)=\omega_{i}^{i}\left(u_{i}, h_{i}\right)
$$

and iii) holds with $u_{i}(t)=0$ and $\phi_{j}^{i}(t)=0$. Therefore, this notion of dissipativity is a natural generalization of the classical one with positive-definite storage functions.

Example 3.6: Let us revisit Examples 3.1 and 3.2. For Example 3.1, we define a class of switching laws

$$
\Pi=\left\{\sigma \mid \begin{array}{rl}
\sigma(x(t), t) & =1 \\
& \Rightarrow\left(\Delta f_{2}\left(x_{1}(t)\right)-\Delta f_{1}\left(x_{1}(t)\right)\right) x_{2}(t) \\
& \leq \exp (-\lambda t) \\
\sigma(x(t), t) & =2 \\
& \Rightarrow\left(\Delta f_{1}\left(x_{1}(t)\right)-\Delta f_{2}\left(x_{1}(t)\right)\right) x_{2}(t) \\
& \leq \exp (-\lambda t) .
\end{array}\right\}
$$

where $\lambda>0$ is any fixed constant.

For any switching law $\sigma \in \prod$, the switched system (4) is dissipative. In fact, i) holds with $\omega_{i}^{i}\left(v_{i}, h_{i}\right)=v_{i} h_{i}$ due to the passivity of each subsystem. ii) has already been shown to be valid in Section III-A. It is easy to check iii) with $v_{i} \equiv 0$ and

$$
\phi_{1}^{2}=\phi_{2}^{1}=\exp (-\lambda t) \in L_{1}^{+}[0, \infty) .
$$

Note that $\prod$ contains infinitely many switching laws because if we set

$$
\Omega_{1}=\left\{x \mid\left(\Delta f_{2}\left(x_{1}\right)-\Delta f_{1}\left(x_{1}\right)\right) x_{2} \leq 0\right\}
$$

and

$$
\Omega_{2}=\left\{x \mid\left(\Delta f_{1}\left(x_{1}\right)-\Delta f_{2}\left(x_{1}\right)\right) x_{2} \leq 0\right\}
$$

then $\Omega_{1} \cup \Omega_{2}=R^{n}$.

In particular, we can pick a state-dependent switching law from $\prod$ as

$$
\sigma(t)=\sigma(x(t))=i \quad \text { if } x(t) \in \Omega_{i}, \quad i=1,2 .
$$

In view of the construction of the cross-supply rates (7) and (10), (23) is the only switching law in $\prod$ that has nonpositive cross-supply rates. 
For Example 3.2, we consider the switching law given by

$$
\begin{aligned}
& \sigma=\sigma(x(t), t) \\
& \left\{\begin{aligned}
1 \quad \text { if } 0 & \leq x_{2}(t)\left(x_{1}(t)-\frac{x_{1}^{3}(t)}{1+x_{2}^{2}(t)}\right) \\
& \leq \exp (-t) \\
\text { or } x_{2}(t)\left(x_{1}(t)-\frac{x_{1}^{3}(t)}{1+x_{2}^{2}(t)}\right) & \\
& <-\frac{1}{1+x_{2}^{2}(t)} \exp (-2 t) \\
2 \quad & \left(x_{1}(t)-\frac{x_{1}^{3}(t)}{1+x_{2}^{2}(t)}\right) \\
& >
\end{aligned}\right.
\end{aligned}
$$

Similarly to the discussion for Example 3.1 earlier, it is easy to verify that the switched system (11) under the switching law (24) is dissipative with Condition iii) that holds for $u_{i}=0$, $\phi_{2}^{1}=e^{-t}$ and $\phi_{1}^{2}=e^{-2 t}$. In addition, the cross-supply rates (12) and (13) are apparently not nonpositive.

\section{Stability Analysis}

This section addresses stability in the sense of Lyapunov. We show how dissipativity can be used to induce stability for the switched system (1).

Theorem 3.7: Under Assumption 2.1, if the system (1) is dissipative with storage functions $S_{i}(x)$ satisfying $S_{i}(0)=0$, then, the origin is stable in the sense of Lyapunov for any control $u_{i}(t)$ satisfying Condition iii).

Proof: For any constant $c>0$, let

$$
\begin{aligned}
& B(c)=\{x \mid\|x\| \leq c\} \\
& r_{i}(c)=\min _{x}\left\{S_{i}(x) \mid\|x\|=c\right\}
\end{aligned}
$$

and

$$
r(c)=\min _{i}\left\{r_{i}(c)\right\}
$$

For any given $\epsilon>0$, we will show that $S_{i}(x(t))<r(\epsilon)$ for any $i \in M$ and $t \geq t_{0}$ provided the initial state $x_{0}$ is in a small neighborhood of the origin. To this end, we will give the proof in three steps:

a). There exists $T>0$, such that for all integer $K$ satisfying $t_{K+1} \geq T$, for any $j \in M, t_{j_{q}} \in \Sigma \mid j$ and $t_{j_{q}}>t_{K+1}$, it holds that

$$
S_{j}\left(x\left(t_{j_{q}}\right)\right)-S_{j}\left(x\left(t_{K+1}\right)\right)<\frac{1}{2} r(\epsilon) .
$$

b). For some integer $K$ satisfying $t_{K+1} \geq T$

$$
S_{i}(x(t))<\frac{1}{2} r(\epsilon)
$$

holds for any $x_{0}$ in some neighborhood of the origin, $t \in$ $\left[t_{0}, t_{K+1}\right)$ and $i \in M$.

c). For the integer $K$ in b), any $j \in M, t_{j_{q}} \in \Sigma \mid j$ and $t_{j_{q}}>$ $t_{K+1}$, we have

$$
S_{j}\left(x\left(t_{j_{q}}\right)\right)<r(\epsilon) .
$$

We first prove a). Condition iii) says that, for $u_{i}$ satisfying

$$
\omega_{i}^{i}\left(u_{i}(t), h_{i}(x(t))\right) \leq 0
$$

there exist $\phi_{j}^{i}(t) \in L_{1}^{+}[0, \infty), i \neq j$, such that

$$
\omega_{j}^{i}\left(x(t), u_{i}(t), h_{i}(x(t)), t\right) \leq \phi_{j}^{i}(t) .
$$

Since $\phi_{j}^{i}(t) \in L_{1}^{+}[0, \infty)$, there exists $T>0$ such that for any $T_{1}, T_{2}, T \leq T_{1} \leq T_{2} \leq \infty$, it holds that

$$
\int_{T_{1}}^{T_{2}} \phi_{j}^{i}(t) d t<\frac{1}{2 m} r(\epsilon), \quad i, j \in M, \quad i \neq j
$$

where $m$ is the number of the subsystems. Now, for any $j \in M$, let $t_{j_{q}} \in \Sigma \mid j$ and $t_{j_{q}}>t_{K+1}$. Obviously, $j_{q} \geq K+2$. It is easy to deduce from (17) and (18) that

$$
\begin{aligned}
S_{j} & \left(x\left(t_{j_{q}}\right)\right)-S_{j}\left(x\left(t_{K+1}\right)\right) \\
& =\sum_{\lambda=1}^{j_{q}-K-1}\left(S_{j}\left(x\left(t_{K+\lambda+1}\right)\right)-S_{j}\left(x\left(t_{K+\lambda}\right)\right)\right) \\
& \leq \sum_{\lambda=1}^{j_{q}-K-1} \int_{t_{K+}}^{t_{K+}}{ }^{\lambda+1} \psi_{j}^{i}{ }^{K+\lambda}(t) d t
\end{aligned}
$$

where

$$
\psi_{j}^{{ }^{i}{ }^{K+} \lambda}(t)=\left\{\begin{array}{l}
\omega_{j}^{j}\left(u_{j}(x(t), t), h_{j}(x(t))\right) \\
\quad \text { if } i_{K+\lambda}=j, \\
{ }^{i}{ }_{j}{ }^{K+} \lambda\left(x(t), u_{{ }_{K+} \lambda}(x(t), t), h_{i_{K+} \lambda}(x(t)), t\right) \\
\quad \text { if } i_{K+\lambda} \neq j .
\end{array}\right.
$$

Note that $\omega_{j}^{j} \leq 0$ and $\omega_{j}{ }^{K}{ }+\lambda \leq \phi_{j}{ }^{K+} \lambda$ by assumption, so we know that $\psi_{j}{ }^{K}{ }^{K+\lambda} \leq \phi_{j}{ }^{K+}+\lambda$.

Taking (25) into account, we have

$$
\begin{aligned}
& S_{j}\left(x\left(t_{j_{q}}\right)\right)-S_{j}\left(x\left(t_{K+1}\right)\right) \\
& \quad \leq \sum_{\lambda=1}^{j_{q}-K-1} \int_{t_{K+} \lambda}^{t}{ }_{K+} \lambda_{+1} \phi_{j}^{i}{ }^{{ }^{K}+\lambda}(t) d t \\
& \quad \leq \sum_{i=1}^{m} \int_{t_{K+1}}^{\infty} \phi_{j}^{i}(t) d t \\
& \quad<\frac{1}{2} r(\epsilon) .
\end{aligned}
$$

We now prove b). Assumption 2.1 says that on the time interval $\left[t_{0}, T\right]$ the system (1) switches at most $K_{T}$ times for some 
$T$-dependent integer $K_{T}$. For notational simplicity, we drop the subscript $T$ and denote $K_{T}$ by $K$. Thus, the $(K+1)$ th switching time $t_{K+1}$ satisfies $t_{K+1} \geq T$, no matter where to start. Note that $S_{i}$ is positive definite and $S_{i}(0)=0$, we can find $\delta_{1}>0, \delta_{1}<\epsilon$, such that $S_{i}(x)<\frac{1}{2} r(\epsilon)$ when $x \in B\left(\delta_{1}\right)$. For this $\delta_{1}>0$, we can find $\delta_{2}>0, \delta_{2}<\delta_{1}$ such that $S_{i}(x)<r\left(\delta_{1}\right)$ when $x \in B\left(\delta_{2}\right)$. Continuing this procedure, we finally have a sequence

$$
\epsilon=\delta_{0}>\delta_{1}>\delta_{2}>\cdots>\delta_{K}>\delta_{K+1}>\delta_{K+2}>0
$$

with the property

$S_{i}(x)<r\left(\delta_{p}\right), \quad$ when $x \in B\left(\delta_{p+1}\right) p=1,2, \ldots, K+1, \forall i$,

$S_{i}(x)<\frac{1}{2} r(\epsilon), \quad$ when $x \in B\left(\delta_{1}\right), \forall i$.

This property is depicted in Fig. 2.

Note that (17) and (20) imply that $S_{i_{k}}(x(t))$ decreases when the $i_{k}$ th subsystem is active, and therefore,

$$
S_{i_{k}}(x(t)) \leq S_{i_{k}}\left(x\left(t_{k}\right)\right), \quad t \in\left[t_{k}, t_{k+1}\right), \quad k=0,1,2, \ldots
$$

In particular, in view of (29), if $x_{0} \in B\left(\delta_{K+2}\right)$, we have

$$
S_{i_{0}}(x(t)) \leq S_{i_{0}}\left(x_{0}\right)<r\left(\delta_{K+1}\right), \quad t \in\left[t_{0}, t_{1}\right) .
$$

We claim that

$$
x(t) \in B\left(\delta_{K+1}\right), \quad t \in\left[t_{0}, t_{1}\right)
$$

holds. In fact, if (32) is not true, there exists $t^{*} \in\left[t_{0}, t_{1}\right)$ such that $x\left(t^{*}\right) \notin B\left(\delta_{K+1}\right)$. Note that $x_{0} \in B\left(\delta_{K+2}\right)$, there must exist $\bar{t} \in\left(t_{0}, t_{1}\right)$ satisfying $\|x(\bar{t})\|=\delta_{K+1}$. According to the definition of $r\left(\delta_{K+1}\right)$, we have $S_{i_{0}}(x(\bar{t})) \geq r\left(\delta_{K+1}\right)$ which contradicts (31).

Obviously, (29), (30), and (32) imply

$$
S_{i_{1}}(x(t)) \leq S_{i_{1}}\left(x\left(t_{1}\right)\right)<r\left(\delta_{K}\right), \quad t \in\left[t_{1}, t_{2}\right)
$$

which, in turn, gives

$$
x(t) \in B\left(\delta_{K}\right), \quad t \in\left[t_{1}, t_{2}\right) .
$$

Recursively repeating these steps, we finally have

$$
S_{i_{K}}(x(t)) \leq S_{i_{K}}\left(x\left(t_{K}\right)\right)<r\left(\delta_{1}\right), \quad t \in\left[t_{K}, t_{K+1}\right)
$$

and

$$
x(t) \in B\left(\delta_{1}\right), \quad t \in\left[t_{K}, t_{K+1}\right) .
$$

By virtue of

$$
\epsilon=\delta_{0}>\delta_{1}>\delta_{2}>\cdots>\delta_{K}>\delta_{K+1}>\delta_{K+2}>0
$$

it holds that

$$
x(t) \in B\left(\delta_{1}\right), \quad t \in\left[t_{0}, t_{K+1}\right) .
$$

Therefore, for any $i \in M=\{1,2, \ldots, m\}$, we have

$$
S_{i}(x(t))<\frac{1}{2} r(\epsilon), \quad t \in\left[t_{0}, t_{K+1}\right) .
$$

Using a) and b) we have

$$
S_{j}\left(x\left(t_{j_{q}}\right)\right) \leq S_{j}\left(x\left(t_{K+1}\right)\right)+\frac{1}{2} r(\epsilon)<r(\epsilon) .
$$

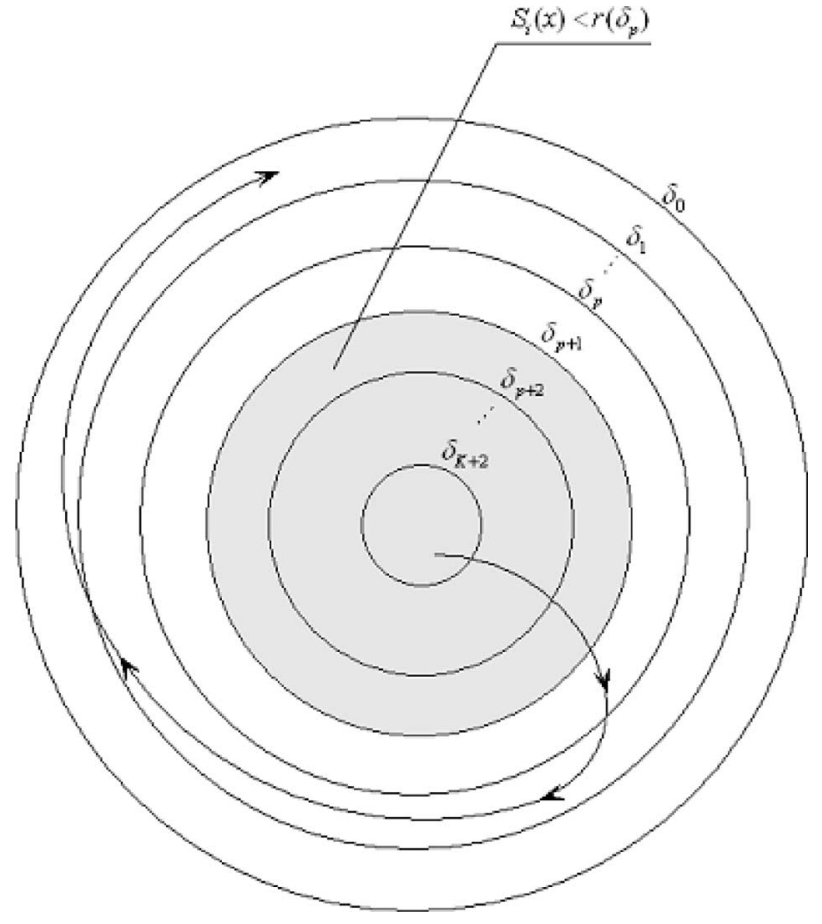

Fig. 2. Proof of Theorem 3.7.

Thus, c) holds. Therefore, it is easy to show that $x(t) \in B(\epsilon)$ for $\forall t$ and stability follows.

Remark 3.8: Normally, stability is addressed for the system (1) with $u_{i}=0$. Here, we consider stability with respect to a specific class of inputs satisfying (19).

Remark 3.9: It can be seen from the proof of Theorem 3.7 that any storage function of an inactive subsystem is allowed to grow but the total growth is bounded by a function in $L_{1}^{+}[0, \infty)$. Moreover, the growth tends to zero as the initial state $x_{0}$ goes to the origin. This is guaranteed by the Assumption 2.1, which means finite switchings in any finite time interval for any initial state $x_{0}$, and by the function $\phi_{j}^{i}$, which is independent of the initial state $x_{0}$.

\section{PAssivity}

Passivity is one of the most useful forms of dissipativity. The connection to stability has a long history reflected in so called Kalman-Yacubovitch-Popov theory (KYP) for linear systems (see [40] and [41]). In this section, we define passivity for system (1) and establish a passivity theorem. We assume that $u_{i}$ and $y_{i}$ have the same dimension.

Definition 4.1: System (1) is said to be passive under the switching law $\Sigma$ if it is dissipative with respect to

$$
\omega_{j}^{j}\left(u_{j}, h_{j}\right)=u_{j}^{T} h_{j}-\delta_{j} u_{j}^{T} u_{j}-\epsilon_{j} h_{j}^{T} h_{j}, \quad j=1,2, \ldots, m
$$

for some $\delta_{j} \geq 0, \epsilon_{j} \geq 0$, and strictly input (output) passive if $\delta_{j}>0\left(\epsilon_{j}>0\right)$. Here, cross-supply rates can take any forms.

Remark 4.2: We only need the supply rates $\omega_{j}^{j}\left(u_{j}, h_{j}\right)$ to be quadratic. Since cross-supply rates, which represent energy exchange between different subsystems, are allowed to take arbitrary forms, this passivity concept is very broad. 
According to this definition, the system (4) in Example 3.1 with any switching law in (22), and the system (11) in Example 3.2 with the switching law (24) are passive.

\section{A. Switched KYP Condition}

We analyze conditions for passivity of switched systems in this section. We focus on smooth affine switched systems of the form

$$
\begin{aligned}
& \dot{x}=f_{\sigma}(x)+g_{\sigma}(x) u_{\sigma} \\
& y=h_{\sigma}(x)
\end{aligned}
$$

with the switching signal $\sigma$ taking values in

$$
M=\{1,2, \ldots, m\} .
$$

We look for smooth storage functions and continuous supply rates and cross-supply rates.

Since for system (39), strict input passivity is never satisfied (see similar discussion on nonswitched systems [39]), we only consider passivity with supply rates

$$
\omega_{i}^{i}\left(u_{i}, h_{i}\right)=u_{i}^{T} h_{i}-\epsilon_{i} h_{i}^{T} h_{i}
$$

We have infinitely many choices of cross-supply rates. In order to generalize the classical KYP condition to switched systems, in this section, for simplicity, we limit ourselves to cross-supply rates of the form

$$
\begin{aligned}
\omega_{j}^{i}\left(x, u_{i}, h_{i}, t\right) & =\varphi_{j}^{i}(x) \omega_{i}^{i}\left(u_{i}, h_{i}\right) \\
& =\varphi_{j}^{i}(x)\left(u_{i}^{T} h_{i}-\epsilon_{i} h_{i}^{T} h_{i}\right)
\end{aligned}
$$

for some positive continuous functions $\varphi_{j}^{i}(x)$.

Also, we only consider two types of switching laws. One is a time-dependent switching law, where $\sigma(t)=i_{k}, t \in\left[t_{k}, t_{k+1}\right)$, and the switching times

$$
\left\{t_{k}, k=1,2, \ldots,\right\}
$$

are predesigned. The other is a state-dependent switching law, where

$$
\sigma(t)=\sigma(x(t))=i \quad \text { if } x(t) \in \Omega_{i}
$$

and

$$
\bigcup_{i=1}^{m} \Omega_{i}=R^{n}, \quad \text { int } \Omega_{i} \bigcap \Omega_{j}=\emptyset, \quad i \neq j .
$$

Note that in these cases, Condition i) and ii) in Definition 3.3 can be written into a unified form

$$
\begin{aligned}
& S_{j}(x(t))-S_{j}(x(s)) \\
& \leq \int_{s}^{t} \varphi_{j}^{i_{k}}(x(\tau)) \\
& \quad \times\left(u_{i_{k}}^{T}(\tau) h_{i_{k}}(x(\tau))-\epsilon_{i_{k}} h_{i_{k}}(x(\tau))^{T} h_{i_{k}}(x(\tau))\right) d \tau \\
& \quad \forall j, k, t_{k} \leq s \leq t<t_{k+1}
\end{aligned}
$$

with $\varphi_{i}^{i}(x)=1$.
For time-dependent switching and state-dependent switching, we have, respectively

$$
\begin{aligned}
& L_{f_{i_{k}}} S_{j} \leq-\epsilon_{i_{k}} \varphi_{j}^{i_{k}} h_{i_{k}}^{T} h_{i_{k}} \quad t \in\left[t_{k}, t_{k+1}\right), \\
& L_{g_{i_{k}}} S_{j}=\varphi_{j}^{i_{k}} h_{i_{k}}^{T}, \quad t \in\left[t_{k}, t_{k+1}\right) .
\end{aligned}
$$

and

$$
\begin{aligned}
& L_{f_{i}} S_{j} \leq-\epsilon_{i} \varphi_{j}^{i} h_{i}^{T} h_{i}, \quad x \in \Omega_{i} \\
& L_{g_{i}} S_{j}=\varphi_{j}^{i} h_{i}^{T}, x \in \Omega_{i}
\end{aligned}
$$

where $L_{f_{i}} S_{j}$ are the Lie derivatives of $S_{j}$ along the vector field $f_{i_{k}}$ while Condition iii) is obviously satisfied due to (40). Inequalities (42) and (43) are localized forms of the well-known passivity (or KYP) conditions [18].

\section{B. Stabilization by Output Feedback}

In this section, we show how passivity induces asymptotic stability via output feedback as in the nonswitched case [19]. Since finite switching is a trivial case, we consider the case of infinite switching.

We first introduce the concept of asymptotic zero-state detectability for nonlinear systems, which will be useful to prove asymptotic stability.

Definition 4.3: A system

$$
\begin{aligned}
& \dot{x}=f(x) \\
& y=h(x)
\end{aligned}
$$

is called asymptotically zero-state detectable if for any $\epsilon>0$, there exists $\delta>0$, such that when $\|y(t+s)\|<\delta$ holds for some $t \geq 0, \Delta>0$ and $0 \leq s \leq \Delta$, we have $\|x(t)\|<\epsilon$.

Remark 4.4: This asymptotic zero-state detectability is a weaker version of small-time norm observability [14].

Theorem 4.5: If the system (1) is passive, then, the origin is stabilized by any controllers $u_{i}$ of the form

$$
u_{i}(t)=\alpha_{i}(x(t), t)
$$

satisfying

$$
f_{i}\left(0, \alpha_{i}(0, t)\right) \equiv 0
$$

and

$$
u_{i}^{T}(t) h_{i}(x(t)) \leq 0 .
$$

If, in addition, all $S_{i}$ are globally defined radially unbounded, there exists at least one $j$ such that

$$
\lim _{k \rightarrow \infty}\left(t_{j_{k}+1}-t_{j_{k}}\right) \neq 0
$$

and all subsystems of (1) are asymptotically zero-state detectable, then, the origin is globally asymptotically stabilizable by output feedback $u_{i}=-h_{i}$.

Proof: Stability follows from Theorem 3.7. The proof of global attractiveness is outlined as follows. We first define a function $\tilde{h}_{j}(t)$ that equals $h_{j}(x(t))$ on infinite many intervals of length no smaller than some positive constant. Then, we show by passivity that $\tilde{h}_{j}(t) \rightarrow 0$ as $t \rightarrow \infty$. Finally, 
we show that $x(t) \rightarrow 0$ as $t \rightarrow \infty$ by asymptotic zero-state detectability.

Substituting the output feedback $u_{i}=-h_{i}$ into the passivity inequality (17) gives rise to

$$
\begin{aligned}
& S_{i_{k}}(x(t))-S_{i_{k}}(x(s)) \\
& \quad \leq-\zeta_{i_{k}} \int_{s}^{t}\left\|h_{i_{k}}(x(t))\right\|^{2} d t, \quad t_{k} \leq s \leq t<t_{k+1}
\end{aligned}
$$

with $\zeta_{i}=1+\delta_{i}+\epsilon_{i}$, or equivalently,

$$
\begin{aligned}
& \zeta_{i_{k}} \int_{s}^{t}\left\|h_{i_{k}}(x(t))\right\|^{2} d t \\
& \quad \leq S_{i_{k}}(x(s))-S_{i_{k}}(x(t)), \quad t_{k} \leq s \leq t<t_{k+1} .
\end{aligned}
$$

For the integer $j$ satisfying

$$
\lim _{k \rightarrow \infty}\left(t_{j_{k}+1}-t_{j_{k}}\right) \neq 0
$$

we can select $\delta>0$ such that the set

$$
\Lambda=\left\{k \mid t_{j_{k}+1}-t_{j_{k}} \geq \delta\right\}
$$

is infinite. Define a function

$$
\tilde{h}_{j}(t)=\left\{\begin{array}{cc}
h_{j}(x(t)), & t \in \bigcup_{k \in \Lambda}\left[t_{j_{k}}, t_{j_{k}+1}\right) \\
0, & \text { otherwise. }
\end{array}\right.
$$

For any $t>0$, if $t_{j_{k}} \leq t<t_{j_{k}+1}$ for some $k \in \Lambda$, (46) gives

$$
\begin{aligned}
& \zeta_{j} \int_{t_{0}}^{t} \tilde{h}_{j}^{T}(t) \tilde{h}_{j}(t) d t \\
& =\zeta_{j} \int_{\left[t_{0}, t\right] \bigcap \bigcup_{p \in \Lambda}\left[t_{j_{p}}, t_{j_{p}+1}\right)} \tilde{h}_{j}^{T}(t) \tilde{h}_{j}(t) d t \\
& =\zeta_{j} \sum_{p \in \Lambda, p<k} \int_{t_{j p}}^{t_{j_{p}+1}} h_{j}^{T}(x(t)) h_{j}(x(t)) d t \\
& +\zeta_{j} \int_{t_{j_{k}}}^{t} h_{j}^{T}(x(t)) h_{j}(x(t)) d t \\
& \leq \zeta_{j} \sum_{p=1}^{k-1} \int_{t_{j_{p}}}^{t_{j_{p}+1}} h_{j}^{T}(x(t)) h_{j}(x(t)) d t \\
& +\zeta_{j} \int_{t_{j_{k}}}^{t} h_{j}^{T}(x(t)) h_{j}(x(t)) d t \\
& \leq \sum_{p=1}^{k-1}\left(S_{j}\left(x\left(t_{j_{p}}\right)\right)-S_{j}\left(x\left(t_{j_{p}+1}\right)\right)\right) \\
& +S_{j}\left(x\left(t_{j_{k}}\right)\right)-S_{j}(x(t)) \\
& \leq \sum_{p=1}^{k}\left(S_{j}\left(x\left(t_{j_{p}}\right)\right)-S_{j}\left(x\left(t_{j_{p}+1}\right)\right)\right) \\
& =S_{j}\left(x\left(t_{j_{1}}\right)\right)-S_{j}\left(t_{j_{k}+1}\right) \\
& +\sum_{p=1}^{k-1}\left(S_{j}\left(x\left(t_{j_{p+1}}\right)\right)-S_{j}\left(x\left(t_{j_{p}+1}\right)\right)\right) .
\end{aligned}
$$

It can be easily derived that

$$
\begin{aligned}
& S_{j}\left(x\left(t_{j_{p+1}}\right)\right)-S_{j}\left(x\left(t_{j_{p}+1}\right)\right) \\
& =\sum_{\lambda=1}^{j_{p+1}-j_{p}-1}\left(S_{j}\left(x\left(t_{j_{p}+1+\lambda}\right)\right)-S_{j}\left(x\left(t_{j_{p}+\lambda}\right)\right)\right) \\
& \leq \sum_{\lambda=1}^{j_{p+1}-j_{p}-1} \\
& \int_{t_{j p+}+\lambda}^{t} t_{j_{p}+1+\lambda}\left(\omega_{j}^{i_{j p}+\lambda}\left(x(t), u_{j_{p}+\lambda}(t), h_{j_{p}+\lambda}(x(t)), t\right)\right) d t \\
& \leq \sum_{\lambda=1}^{j_{p+1}-j_{p}-1} \int_{t_{j p+}+\lambda}^{t_{j_{p}+1+\lambda}} \phi_{j}^{i{ }_{j p+} \lambda}(t) d t .
\end{aligned}
$$

Therefore,

$$
\begin{aligned}
& \sum_{p=1}^{k-1}\left(S_{j}\left(x\left(t_{j_{p+1}}\right)\right)-S_{j}\left(x\left(t_{j_{p}+1}\right)\right)\right.
\end{aligned}
$$

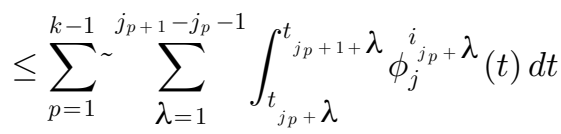

$$
\begin{aligned}
& =\sum_{\lambda=1}^{j_{p+1}-j_{p}-1} \sum_{p=1}^{k-1} \int_{t_{j p}+\lambda}^{t_{j p+1+} \lambda} \phi_{j}^{{ }_{j}{ }_{j p}+\lambda}(t) d t \\
& \leq \sum_{i=1, i \neq j}^{m} \int_{t_{0}}^{\infty} \phi_{j}^{i}(t) d t \\
& <\infty \text {. }
\end{aligned}
$$

If $t \notin\left[t_{j_{k}}, t_{j_{k}+1}\right)$ for any $k \in \Lambda$, then there exists $k \in \Lambda$ such that $t \geq t_{j_{k}+1}$ and $t<t_{j_{q}}$ for any $q \in \Lambda$ and $q>k$. In this case, we have

$$
\tilde{h}_{j}(s) \equiv 0, \quad s \in\left[t_{j_{k}+1}, t\right]
$$

and (48) still holds. It follows from (48) and (50) that $\int_{t_{0}}^{\infty} \tilde{h}_{j}^{T}(t) \tilde{h}_{j}(t) d t$ is finite. Now, we show $\tilde{h}_{j}(t) \rightarrow 0$ as $t \rightarrow \infty$. Suppose this is false; then there exist $\epsilon>0$ and a sequence of time $t$, say,

$$
q_{1}, q_{2}, \ldots, q_{k} \rightarrow \infty
$$

satisfying

$$
\tilde{h}_{j}^{T}\left(q_{i}\right) \tilde{h}_{j}\left(q_{i}\right) \geq \epsilon, \quad \forall i .
$$

Note that (45) and Condition ii) in Definition 3.3 guarantee the boundedness of $x(t)$, and

$$
\dot{x}(t)=f_{\sigma}(x(t))
$$

is also bounded. Hence, $\tilde{h}_{j}(t)$ is uniformly continuous over

$$
\bigcup_{k \in \Lambda}\left[t_{j_{k}}, t_{j_{k}+1}\right) \text {. }
$$

In view of

$$
t_{j_{k}+1}-t_{j_{k}} \geq \delta, \quad k \in \Lambda
$$




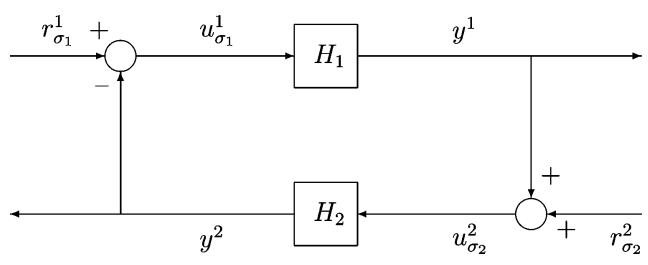

Fig. 3. Feedback interconnection.

we have

$$
\int_{t_{0}}^{\infty} \tilde{h}_{j}^{T}(t) \tilde{h}_{j}(t) d t=\infty
$$

which contradicts the fact that $\int_{t_{0}}^{\infty} \tilde{h}_{j}^{T}(t) \tilde{h}_{j}(t) d t$ is finite. Therefore, $\tilde{h}_{j}(t) \rightarrow 0$. So, $x\left(t_{j_{k}}\right) \rightarrow 0$ as $k \rightarrow \infty$ and $k \in \Lambda$ follows from the asymptotic zero-state detectability of the $j$ th subsystem. This, in turn, implies $x(t) \rightarrow 0$ as $t \rightarrow \infty$ due to stability of the closed-loop system and continuity of $x(t)$.

Remark 4.6: The control $u_{i}$ can be chosen as output feedback of the form $u_{i}=-\xi_{i}\left(h_{i}\right)$ satisfying $\xi_{i}^{T}(y) y>0$ for any $y$.

Remark 4.7: If the system (1) is strict output passive, global asymptotic stability follows for $u_{i}=0$. The proof is similar.

\section{Feedback Interconnection}

From the nonswitched system theory [17], [40], passivity is expected to be preserved under feedback interconnection. This property is very useful in stability analysis of interconnected systems. In this section, we give the feedback interconnection theorem for passive switched systems.

Consider the switched systems

$$
\begin{aligned}
H_{1}: \quad \dot{x}^{1} & =f_{\sigma_{1}}^{1}\left(x^{1}, u_{\sigma_{1}}^{1}\right) \\
y^{1} & =h_{\sigma_{1}}^{1}\left(x^{1}\right)
\end{aligned}
$$

with $x^{1} \in R^{n_{1}}$ and the switching signal $\sigma_{1}$, and

$$
\begin{aligned}
H_{2}: & \dot{x}^{2}=f_{\sigma_{2}}^{2}\left(x^{2}, u_{\sigma_{2}}^{2}\right) \\
& y^{2}=h_{\sigma_{2}}^{2}\left(x^{2}\right)
\end{aligned}
$$

with $x^{2} \in R^{n_{2}}$ and the switching signal $\sigma_{2}$. We assume that $f_{\sigma_{i}}^{i}$ and $h_{\sigma_{i}}^{i}$ are continuous. The feedback interconnection of $H_{1}$ and $H_{2}$ is depicted in Fig. 3.

Theorem 4.8: Suppose that the switched systems $H_{1}$ and $H_{2}$ are passive. Then, the feedback interconnected system shown in Fig. 3 is again a passive switched system as long as the corresponding interconnected subsystems are compatible in the sense of dimensions, i.e., at any time $t$, it must hold that

$$
\begin{aligned}
& \operatorname{dim} r_{\sigma_{1}}^{1}=\operatorname{dim} h_{\sigma_{2}}^{2}=\operatorname{dim} u_{\sigma_{1}}^{1} \\
& \operatorname{dim} r_{\sigma_{2}}^{2}=\operatorname{dim} h_{\sigma_{1}}^{1}=\operatorname{dim} u_{\sigma_{2}}^{2} .
\end{aligned}
$$

Furthermore, if both $H_{1}$ and $H_{2}$ are strictly output passive, then the interconnected system is also strictly output passive.
Proof: Similar to the nonswitched case [39], it is therefore, omitted here.

\section{V. $L_{2}$-GAIN}

As another useful form of dissipativity, we study $L_{2}$-gain of switched systems in this section.

Definition 5.1: The system (1) is said to have $L_{2}$-gain $\gamma>0$ if it is dissipative with respect to

$$
\omega_{i}^{i}=\frac{1}{2} \gamma^{2} u_{i}^{T} u_{i}-\frac{1}{2} h_{i}^{T} h_{i}, \quad i=1,2, \cdots, m .
$$

Similarly to the definition of passivity, we do not specify the form of cross-supply rates $\omega_{i}^{j}, i \neq j$.

\section{A. Switched Hamilton-Jacobi Inequalities}

We now look for conditions under which the system (1) has $L_{2}$-gain. Again, in this section, we focus on the smooth affineswitched system (39) with cross-supply rates of the form

$$
\begin{aligned}
\omega_{j}^{i}\left(x, u_{i}, h_{i}, t\right) & =\varphi_{j}^{i}(x) \omega_{i}^{i}\left(u_{i}, h_{i}\right) \\
& =\frac{1}{2} \varphi_{j}^{i}(x)\left(\gamma^{2} u_{i}^{T} u_{i}-h_{i}^{T} h_{i}\right)
\end{aligned}
$$

for some positive continuous functions $\varphi_{j}^{i}(x)$. Similarly to (41), Conditions i) and ii) in Definition 3.3 can be written into a unified form

$$
\begin{aligned}
& S_{j}(x(t))-S_{j}(x(s)) \\
& \quad \leq \frac{1}{2} \int_{s}^{t} \varphi_{j}^{i_{k}}(x(\tau)) \\
& \quad \times\left(\gamma^{2} u_{i_{k}}^{T}(\tau) u_{i_{k}}(\tau)-h_{i_{k}}(x(\tau))^{T} h_{i_{k}}(x(\tau))\right) d \tau \\
& \quad \forall j, k, t_{k} \leq s \leq t<t_{k+1}
\end{aligned}
$$

with $\varphi_{i}^{i}(x)=1$. Following a similar way as in [39], we have the switched Hamilton-Jacobi inequalities, respectively, for timedependent switching and state-dependent switching as

$$
\begin{aligned}
& L_{f_{i_{k}}} S_{j}+\frac{1}{2 \varphi_{j}^{i_{k}} \gamma^{2}}\left(L_{g_{i_{k}}} S_{j}\right)\left(L_{g_{i_{k}}} S_{j}\right)^{T}+\frac{1}{2} \varphi_{j}^{i_{k}} h_{i_{k}}^{T} h_{i_{k}} \\
& \quad \leq 0, t \in\left[t_{k}, t_{k+1}\right)
\end{aligned}
$$

and

$$
\begin{aligned}
& L_{f_{i}} S_{j}+\frac{1}{2 \varphi_{j}^{i} \gamma^{2}}\left(L_{g_{i}} S_{j}\right)\left(L_{g_{i}} S_{j}\right)^{T}+\frac{1}{2} \varphi_{j}^{i} h_{i}^{T} h_{i} \\
& \quad \leq 0, x \in \Omega_{i} .
\end{aligned}
$$

Equations (55) and (56) are localized forms of the HamiltonJacobi inequalities that generalize those used in nonswitched systems [18], [39].

\section{B. Stabilization}

As in nonswitched systems, $L_{2}$-gain can produce stability for a certain class of control signals. Again, we only consider the nontrivial case of infinite switching. 
Theorem 5.2: If the system (1) has $L_{2}$-gain $\gamma$, then the origin is stabilized by any control $u_{i}(t)$ satisfying

$$
\left\|u_{i}(t)\right\|^{2} \leq \frac{\left(1-\zeta_{i}^{2}\right)}{\gamma^{2}}\left\|h_{i}(t)\right\|^{2}
$$

for some $\zeta_{i}, 0 \leq \zeta_{i} \leq 1$. If in addition, $0<\zeta_{i} \leq 1$, all $S_{i}$ are globally defined radially unbounded, there exists at least one $j$ such that

$$
\lim _{k \rightarrow \infty}\left(t_{j_{k}+1}-t_{j_{k}}\right) \neq 0
$$

and all subsystems of the system (1) are asymptotically zero-state detectable, then the origin is globally asymptotically stabilized.

Proof: Similar to the proof of Theorem 4.5.

\section{Small-Gain Theorem}

This section establishes a small-gain theorem for switched systems.

Suppose we have two switched systems:

$$
\begin{aligned}
H_{1}: \quad \dot{x} & =f_{\sigma_{1}}\left(x, u_{\sigma_{1}}\right) \\
& y=h_{\sigma_{1}}(x)
\end{aligned}
$$

and

$$
\begin{aligned}
\quad \dot{z} & =g_{\sigma_{2}}\left(z, v_{\sigma_{2}}\right) \\
H_{2}: \quad & =l_{\sigma_{2}}(z)
\end{aligned}
$$

where

$$
\sigma_{i}: R_{+} \rightarrow M_{i}=\left\{1,2, \ldots, m_{i}\right\}, i=1,2 .
$$

The meaning of other variables are the same as those in the system (1). Without loss of generality, we assume that the two switched systems have the same switching time sequence

$$
\left\{t_{0}, t_{1}, \ldots, t_{k}, \ldots\right\} \text {. }
$$

When $t \in\left[t_{k}, t_{k+1}\right)$, the $i_{k}^{1}$ th and $i_{k}^{2}$ th subsystems of $H_{1}$ and $\mathrm{H}_{2}$ are active, respectively.

Theorem 5.3: Suppose that $H_{1}$ has $L_{2}$-gain $\gamma_{1}$ with storage functions $S_{1 i}$ and cross-supply rates $\omega_{1 j}^{i}$, and $H_{2}$ has $L_{2}$-gain $\gamma_{2}$ with storage functions $S_{2 i}$ and cross-supply rates $\omega_{2 j}^{i}$, respectively. If $\gamma_{1} \gamma_{2}<1$, and

$$
\begin{aligned}
& \omega_{1 j}^{i}\left(x(t), u_{i}(t), h_{i}(x(t)), t\right) \\
& \quad \leq \frac{1}{2} C(t)\left(\gamma_{1}^{2} u_{i}^{T}(t) u_{i}(t)-h_{i}^{T}(x(t)) h_{i}(x(t))\right)+\varphi_{1 j}^{i}(t) \\
& \omega_{2 j}^{i}\left(z(t), v_{i}(t), l_{i}(x(t)), t\right) \\
& \quad \leq \frac{1}{2} C(t)\left(\gamma_{2}^{2} v_{i}^{T}(t) v_{i}(t)-l_{i}^{T}(z(t)) l_{i}(z(t))\right)+\varphi_{2 j}^{i}(t)
\end{aligned}
$$

for some nonnegative function $C(t)$, functions $\varphi_{1 j}^{i}(t), \varphi_{2 j}^{i} \in$ $L_{1}^{+}[0, \infty)$, then, the feedback-interconnected system of $H_{1}$ and $\mathrm{H}_{2}$ with

$$
u_{\sigma_{1}}=-l_{\sigma_{2}}(z), v_{\sigma_{2}}=h_{\sigma_{1}}(x)
$$

is stable. If in addition, all $S_{1 i}, S_{2 i}$ are globally defined radially unbounded, there exists at least one interconnection of subsystems having infinite active time intervals with positive dwell time, and all subsystems of $H_{1}$ and $H_{2}$ are asymptotically zero state detectable, then the feedback interconnected system is globally asymptotically stable.

Proof: The conclusion can be proven by applying the method in [19] and [39].

Remark 5.4: It is not surprising that in addition to the usual small-gain condition $\gamma_{1} \gamma_{2}<1$, we still need the condition (60), which is a constraint on cross-supply rates. This constraint can be regarded as a generalization of the small-gain condition between inactive coupled subsystems. In particular, when crosssupply rates are the same as supply rates, this condition is automatically satisfied. In general, the cross-supply rates are allowed to take any form.

\section{CONCLUding REMARK}

We have established a framework of dissipativity theory for switched systems. Multiple storage functions and multiple supply rates are adopted to describe dissipativity. When a subsystem is active, the dissipativity property is consistent with the classical notion of dissipativity for continuous systems. For inactive subsystems, the change of associated storage functions, viewed as "exchanged energy" from the active subsystem to inactive subsystems, is measured by cross-supply rates. Stability is assured by dissipativity. For passivity, we localize the passivity (or Kalman-Yacubovitch-Popov) condition and derive a property of invariance under feedback interconnection. For $L_{2}$-gain, we give a local version of the Hamilton-Jacobi inequalities and study the small-gain property.

The introduction of cross-supply rates relates the active subsystem and inactive subsystems. As in the classic notion of dissipativity, where the supply rate may represent abstract energy, cross-supply rates in dissipativity of switched systems are abstract "exchanged energy." Therefore, we do not limit the form of cross-supply rates in the description of dissipativity, passivity, and $L_{2}$-gain in order to cover more general situations. In particular, cross-supply rates are allowed to be positive even though it must be possible for all supply rates to be negative, which completely differs from the classical dissipativity property. Moreover, a storage function is allowed to grow on the "switched ON" time sequence of the corresponding subsystem. This feature makes the dissipativity theory established here different from the Branicky's theory of multiple Lyapunov functions [2], in which the nonincreasing condition of a Lyapunov function on the "switched ON" time sequence is a key prerequisite. It is worthwhile pointing out that sometimes the general cross-supply rates may "weaken" the results. For example, the $L_{2}$-gain property only describes the inputoutput gain over the active time intervals. In order to have the input-output gain over the infinite time domain, which is related to $H_{\infty}$ control, certain constraints must be imposed on the cross-supply rates. This will be considered in our separate papers.

An important research problem is dissipation via feedback. The general form of cross-supply rates provides more possibility for dissipation. A potential application of the dissipativity theory proposed in this paper is to stabilize continuous-time 
nonlinear systems via controller switching. This needs further research. Other results will more fully generalize the theory for nonswitched systems, e.g., feedback passivity theorems [19].

\section{REFERENCES}

[1] A. Bemporad, G. Bianchini, F. Brogi, and F. Barbagli, "Passivity analysis and passification of discrete-time hybrid systems," in Proc. Preprints 16th IFAC World Congr., Prague, Czech Republic, Jul. 2005, pp. 151-167, (CD Rom).

[2] M. S. Branicky, "Multiple Lyapunov functions and other analysis tools for switched and hybrid systems," IEEE Trans. Autom. Control, vol. 43, no. 4, pp. 475-482, Apr. 1998.

[3] C. I. Byrnes, A. Isidori, and J. C. Willems, "Passivity feedback equivalence, and the global stabilization of minimum phase nonlinear systems," IEEE Trans. Autom. Control, vol. 36, no. 11, pp. 1228-1240, Nov. 1991.

[4] W. Chen and M. Saif, "Passivity and passivity based controller design of a class of switched control systems," in Proc. Preprints 16th IFAC World Congr., Prague, Czech Republic, Jul. 2005, pp. 143-147, (CD Rom).

[5] R. A. Decarlo, M. S. Branicky, S. Pettersson, and B. Lennartson, "Perspectives and results on the stability and stabilization of hybrid systems," Proc. IEEE, vol. 88, no. 7, pp. 1069-1082, Jul. 2000.

[6] G. Escobar, R. Ortega, H. Sira-Ramirez, and H. Ludvigsen, "A hybrid passivity based controller design for a three phase voltage sourced reversible boost type rectifier," in Proc. 37th IEEE Conf. Decis. Control, 1998, pp. 2035-2040.

[7] N. H. EI-Farra, P. Mhaskar, and P. D. Christofides, "Output feedback control of switchied nonlinear systems using multiple Lyapunov functions," Syst. Control Lett., vol. 54, pp. 1163-1182, 2005.

[8] W. M. Haddad and V. Chellaboina, "Dissipativity theory and stability of feedback interconnections for hybrid dynamical systems," Math. Probl. Eng., vol. 7, pp. 299-335, 2001.

[9] W. M. Haddad, V. Chellaboina, and N. A. Kablar, "Nonlinear impulsive dynamical systems. Part I: Stability and dissipativity," Probl. Int. J. Control, vol. 74, pp. 1631-1658, 2001.

[10] W. M. Haddad, V. Chellaboina, Q. Hui, and S. Nersesov, "Vector dissipativity theory for large-scale impulsive dynamical systems," Math. Probl. Eng., vol. 10, pp. 225-262, 2004.

[11] W. M. Haddad, V. Chellaboina, Q. Hui, and S. G. Nersesov, "Thermodynamic stabilization via energy dissipating hybrid controllers," in Proc. 44th IEEE Conf. Decis. Control Eur. Control Conf., Seville, Spain, 2005, pp. 4879-4884.

[12] A. Hassibi and S. Boyd, "Quadratic stabilization and control of piecewiselinear systems," in Proc. Decis. Amer. Control Conf., Philadelphia, PA, 1998, pp. 3659-3664.

[13] J. W. Helton and M. R. James, Extending $H_{\infty}$ Control to Nonlinear Systems. Philadelphia, PA: SIAM, 1999.

[14] J. P. Hespanha, D. Liberzon, D. Angeli, and E. D. Sontag, "Nonlinear norm observability notions and stability of switched systems," IEEE Trans. Autom. Control, vol. 50, no. 2, pp. 154-168, Feb. 2005.

[15] J. P. Hespanha and A. S. Morse, " $L_{2}$-induced gains of switched linear systems," in Open Problems in Mathematical Systems Theory and Control, V. D. Blondel, E. D. Sontag, M. Vidyasagar, and J. C. Willems, Eds. London, U.K.: Springer-Verlag, 1999, pp. 45-47.

[16] J. P. Hespanha, "Root-mean-square gains of switched linear systems theory," IEEE Trans. Autom. Control, vol. 48, no. 11, pp. 2040-2045, Nov. 2003.

[17] D. J. Hill and P. J. Moylan, "Dissipative dynamical systems: Basic inputoutput and state properties," J. Franklin Inst., vol. 309, pp. 327-357, 1980.

[18] D. J. Hill and P. J. Moylan, "The stability of nonlinear dissipative systems," IEEE Trans. Autom. Control, vol. AC-21, no. 5, pp. 708-711, Oct. 1976.

[19] D. J. Hill and P. J. Moylan, "Stability results for nonlinear feedback systems," Automatica, vol. 13, pp. 377-382, 1977.

[20] I. V. Kolmanovsky and J. Sun, "A multi-mode switching-based command tracking in network controlled systems with pointwise-in-time constraints and disturbance inputs," in Proc. 6th WCICA, 2006, pp. 199104.
[21] A. Leonessa, W. M. Haddad, and V. Chellaboina, "Nonlinear system stabilization via hierarchical switching control," IEEE Trans. Autom. Control, vol. 46, no. 1, pp. 17-28, Jan. 2001.

[22] Z. Li, Y. Soh, and C. Wen, Switched and Impulsive Systems: Analysis, Design and Applications. Berlin, Germany: Springer-Verlag, 2005.

[23] D. Liberzon and A. S. Morse, "Basic problems in stability and design of switched systems," IEEE Control Syst. Mag., vol. 19, no. 5, pp. 59-70, Oct. 1999.

[24] D. Liberzon, Switching in Systems and Control. Boston, MA: Birkhauser, 2003.

[25] H. Lin and P. J. Antsaklis, "Stability and stabilizability of switched linear systems: A short survey of recent results," in Proc. 44th IEEE Conf. Decis. Control, Eur. Control Conf. 2005, Seville, Spain, pp. 24-29.

[26] R. Lozano, B. Brogliato, O. Egeland, and B. Maschke, Dissipative Systems Analysis and Control. London, U.K.: Springer-Verlag, 2000.

[27] P. Mhaskar, N. H. EI-Farra, and P. D. Christofides, "Predictive control of switched nonlinear systems with scheduled mode transitions," IEEE Trans. Autom. Control, vol. 50, no. 11, pp. 1670-1680, Nov. 2005

[28] A. N. Michel and B. Hu, "Towards a stability theory of general hybrid dynamical systems," Automatica, vol. 35, pp. 371-384, 1999.

[29] K. S. Narendra, O. A. Driollet, M. Feiler, and K. George, "Adaptive control using multiple models, switching and tuning," Int.J. Adapt. Control Signal Process., vol. 17, pp. 87-102, 2003.

[30] T. Ooba and Y. Funahashi, "On a common quadratic Lyapunov function for widely distant systems," IEEE Trans. Autom. Control, vol. 42, no. 12, pp. 1697-1699, Dec. 1997.

[31] P. Peleties and R. DeCarlo, "Asymptotic stability of $m$-switched systems using Lyapunov-like functions," in Proc. Amer. Control Conf., 1991, pp. 1679-1684

[32] G. E. Perez, P. M. Ortiz, M. V. Villa, and H. S. Ramirez, "Passivity-based control of switched reluctance motors with nonlinear magnetic circuits," IEEE Trans. Control Syst. Technol., vol. 12, no. 3, pp. 439-448, May 2004.

[33] A. Y. Pogromsky, M. Jirstrand, and P. Spangeus, "On stability and passivity of a class of hybrid systems," in Proc. 37th IEEE Conf. Decis. Control, 1998, pp. 3705-3710.

[34] I. G. Polushin and H. J. Marquez, "On the existence of a continuous storage function for dissipative systems," Syst. Control Lett., vol. 46, pp. 85-90, 2002.

[35] C. A. Schwartz and E. Maben, "A minimum energy approach to switching control for mechanical systems," in Control Using Logic-Based Switching, A. S. Morse, Ed. New York: Springer-Verlag, 1997, pp. 142-150.

[36] E. Skafidas, R. J. Evans, A. V. Savkin, and I. R. Petersen, "Stability results for switched controller systems," Automatica, vol. 35, pp. 553-564, 1999.

[37] X. Sun, J. Zhao, and D. J. Hill, "Stability and $L_{2}$-gain analysis for switched delay systems: A delay-dependent method," Automatica, vol. 42, pp. 1769-1774, 2006.

[38] Z. Sun and S. S. Ge, Switched Linear Systems-Control and Design. London, U.K.: Springer-Verlag, 2005.

[39] A. J. van der Schaft, $L_{2}$-Gain and Passivity Techniques in Nonlinear Control. London, U.K.: Springer-Verlag, 2000.

[40] J. C. Willems, "Dissipative dynamical systems, part I: General theory," Arch. Ration. Mech. Anal., vol. 45, pp. 321-351, 1972.

[41] J. L. Wyatt Jr., L. O. Chua, J. W. Gannett, I. C. Goknar, and D. N. Green, "Energy concepts in the state-space theory of nonlinear $n$-parts: Part onePassivity," IEEE Trans. Circuits Syst., vol. CAS-28, no. 1, pp. 48-61, Jan. 1981.

[42] M. Zefran, F. Bullo, and M. Stein, "A notion of passivity for hybrid systems," in Proc. 40th IEEE Conf. Decis. Control, 2001, pp. 768-773.

[43] G. Zhai, B. Ho, K. Yasuda, and A. N. Michel, "Disturbance attenuation properties of time-controlled switched systems," J. Franklin Inst., vol. 338, pp. 765-779, 2001.

[44] J. Zhao and G. M. Dimirovski, "Quadratic stability of a class of switched nonlinear systems," IEEE Trans. Autom. Control, vol. 49, no. 4, pp. 574 578, Apr. 2004.

[45] J. Zhao and D. J. Hill, "Hybrid $H_{\infty}$ control based on multiple Lyapunov functions," in Proc. 6th IFAC Symp. Nonlinear Control Syst., 2004, pp. 567-572.

[46] J. Zhao and D. J. Hill, "A notion of passivity for switched systems with state-dependent switching," J. Control Theory Appl., vol. 4, pp. 70-75, 2006.

[47] J. Zhao and M. W. Spong, "Hybrid control for global stabilization of the cart-pendulum system," Automatica, vol. 37, pp. 1941-1951, 2001. 


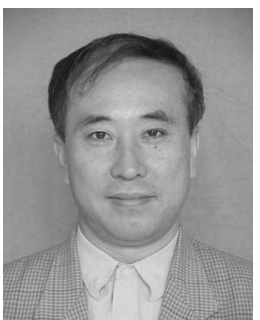

Jun Zhao received the B.S. and M.S. degrees in mathematics from Liaoning University, Shenyang, China, in 1982 and 1984, respectively, and the Ph.D. degree in control theory and applications from the Northeastern University, Shenyang, in 1991.

From 1992 to 1993, he was a Postdoctoral Fellow at the Northeastern University, where since 1994, he has been with the School of Information Science and Engineering, and is currently a Professor. From February 1998 to February 1999, he was a Senior Visiting Scholar at the Coordinated Science Laboratory, University of Illinois, Urbana-Champaign. From November 2003 to May 2005, he was a Research Fellow in the Department of Electronic Engineering, City University of Hong Kong. Since November 2006, he has also been with the Department of Information Engineering, Research School of Information Sciences and Engineering, The Australian National University, Canberra, A.C.T., Australia, as a Fellow. His current research interests include switched systems, hybrid control, nonlinear systems, and robust control.

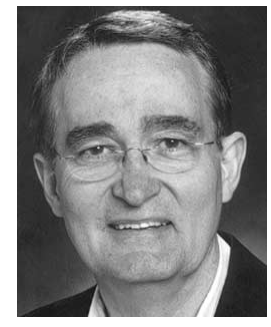

David J. Hill (M'76-SM'91-F'93) received the B.E. degree in electrical engineering and B.Sc. degree in mathematics from the University of Queensland, Brisbane, QLD, Australia, in 1972 and 1974, respectively, and the Ph.D. degree in electrical engineering from the University of Newcastle, Callaghan, N.S.W., Australia, in 1976.

$\mathrm{He}$ is currently an Australian Research Council Federation Fellow in the Research School of Information Sciences and Engineering, The Australian National University, Canberra, A.C.T., Australia. He held academic and substantial visiting positions at the Universities of Melbourne, California (Berkeley), Newcastle (Australia), Lund (Sweden), Sydney, and Hong Kong (City University). He holds honorary professorships at the University of Sydney, the University of Queensland (Australia), South China University of Technology, City University of Hong Kong, Wuhan University, and Northeastern University (China). His current research interests include network systems science, stability analysis, nonlinear control and applications.

Dr. Hill is a Fellow of the Institution of Engineers, Australia, and the Australian Academy of Science. He is also a Foreign Member of the Royal Swedish Academy of Engineering Sciences. 